\title{
Anestesia regional como alternativa para procedimentos médicos na pandemia da COVID-19
}

\author{
Regional anesthesia as alternative to medical procedures in the COVID-19 pandemic \\ La anestesia regional como alternativa a los procedimientos médicos en la pandemia de COVID-19
}

\section{Resumo}

A pandemia de coronavírus (SARS-CoV-2) impactou significativamente os sistemas de saúde e desafiou a equipe multidisciplinar, sendo considerado um problema de saúde pública global. Diversas estratégias foram sugeridas visando a segurança e minimizar a exposição dos profissionais de saúde e pacientes hospitalizados ao vírus, sendo uma destas evitar procedimentos geradores de aerossóis durante os procedimentos cirúrgicos, como o manejo das vias aéreas. Diante disto, a anestesias regional e local tornaram-se como uma alternativa viável, eficaz e segura a anestesia geral, desempenhando um papel importante durante o surto da COVID-19, quando aplicável. Dentre as vantagens pode-se citar a não geração de aerossóis, analgesia pós-operatória, redução de náuseas e vômitos e também, de complicações cardiorrespiratórias. Este estudo objetiva investigar a aplicação da anestesia regional nas diversas especialidades médicas durante a pandemia de coronavírus avaliando os resultados do emprego desta. Foi realizada uma busca nas bases de dados Pubmed, Web of Science (todas as bases de dados), Scopus, Embase e Cochrane Library utilizando os termos de busca "SARS-CoV-2", "COVID-19", "Anesthesia, Local" e "Anesthetics, Local" onde 49 referências foram incluídas. Os estudos demonstram bons resultatos nas técnicas empregadas e possibilitam uma alternativa de tratamento em diversos campos cirúrgicos de maneira segura. Dessa forma a anestesias regional e local são adequadas e viáveis para procedimentos médicos cirúrgicos e constituem uma boa alternativa a anestesia geral podendo ser aplicadas em diferentes especialidades cirúrgicas.

Palavras-chave: Anestesia local; Anestésicos locais; COVID-19; SARS-CoV-2.

\begin{abstract}
The coronavirus pandemic (SARS-CoV-2) significantly impacted health systems and challenged the multidisciplinary team, which is considered a global public health problem. Several strategies have been suggested aiming at safety and minimizing the exposure of health professionals and hospitalized patients to the virus, one of which is to avoid procedures that generate aerosols during surgical procedures, such as airway management. Therefore, regional and local anesthesia has become a viable, effective and safe alternative to general anesthesia, playing an important role during the outbreak of COVID-19, when applicable. Among the advantages, one can mention the non-generation of aerosols, postoperative analgesia, reduction of nausea and vomiting, as well as cardiorespiratory complications. This study aims to investigate the application of regional anesthesia in different medical specialties during the coronavirus pandemic, evaluating the results of its use. A search was performed in Pubmed, Web of Science (all databases), Scopus, Embase, and Cochrane Library databases using the search terms "SARS-CoV-2", "COVID-19", "Anesthesia, Local" and "Anesthetics, Local" where 49 references were included. Studies show good results in the techniques used and enable a safe alternative treatment in various surgical fields. Thus, regional and local anesthesia are suitable and viable for medical surgical procedures and constitute a good alternative to general anesthesia and can be applied in different surgical specialties.
\end{abstract}

Keywords: Anesthesia, local; Anesthetics, local; COVID-19; SARS-CoV-2. 


\section{Resumen}

La pandemia de coronavirus (SARS-CoV-2) afectó significativamente los sistemas de salud y desafió al equipo multidisciplinario, que se considera un problema de salud pública mundial. Se han sugerido varias estrategias orientadas a la seguridad y minimizar la exposición al virus de los profesionales de la salud y de los pacientes hospitalizados, una de las cuales es evitar procedimientos que generen aerosoles durante los procedimientos quirúrgicos, como el manejo de la vía aérea. Por lo tanto, la anestesia regional y local se ha convertido en una alternativa viable, eficaz y segura a la anestesia general, desempeñando un papel importante durante el brote de COVID-19, cuando corresponde. Entre las ventajas, se pueden mencionar la no generación de aerosoles, analgesia postoperatoria, reducción de náuseas y vómitos, así como complicaciones cardiorrespiratorias. Este estudio tiene como objetivo investigar la aplicación de anestesia regional en diferentes especialidades médicas durante la pandemia de coronavirus, evaluando los resultados de su uso. Se realizó una búsqueda en las bases de datos de Pubmed, Web of Science (todas las bases de datos), Scopus, Embase y Cochrane Library utilizando los términos de búsqueda "SARSCoV-2", "COVID-19", "Anesthesia, Local" y "Anesthetics, Local" donde se incluyeron 49 referencias. Los estudios muestran buenos resultados en las técnicas utilizadas y permiten un tratamiento alternativo seguro en diversos campos quirúrgicos. Así, la anestesia regional y local son aptas y viables para procedimientos médico-quirúrgicos y constituyen una buena alternativa a la anestesia general y pueden aplicarse en diferentes especialidades quirúrgicas.

Palabras clave: Anestesia local; Anestésicos locales; COVID-19; SARS-CoV-2.

\section{Introdução}

A pandemia do SARS-CoV-2 (COVID-19) constitui uma emergência global de saúde pública com altas taxas de mortalidade, desafiando a equipe multiprofissional e sistemas de saúde em todo o mundo, com velocidades de reação, diferentes tipos de resposta e desconhecimento de algumas características virais (Coulthard, 2020; Sabino-silva et al., 2020). Esta condição expõe os profissionais de saúde a grande risco de contaminação nosocomial, reforçando a necessidade da adoção de medidas preventivas de forma rigorosa (Moreira et al., 2020).

Neste contexto, a anestesiologia enfrentou vários desafios, onde a viabilidade e segurança do manejo anestésico em pacientes com suspeita ou infectados pelo SARS-CoV-2 passaram a ser questionadas, levando em consideração o risco de exposição e contaminação dos profissionais de saúde e demais pacientes hospitalizados. Ademais, os fármacos administrados para o tratamento e as alterações fisiopatológicas da COVID-19 podem provocar desfechos inesperados e até mesmo, desfavoráveis pela interação com os anestésicos e/ou técnicas anestésicas (Moreira et al., 2020).

Diante disto, a Association of Anesthetists e o Royal College of Anesthetists orientaram potenciais mitigações para a situação, como o oferecimento de fármacos e técnicas alternativas, dentre elas o uso da anestesia local e regional quando praticável e seguro em alternativa a anestesia geral (Association of Anesthetists, Royal College of Anesthetists, 2020).

Diante das novas necessidades da literatura, o presente estudo busca revisar a literatura atual a respeito do uso de anestésicos locais como alternativa ao manejo de pacientes durante a pandemia de COVID-19, evidenciando sua possibilidade de uso de modo a garantir a segurança para os profissionais de centros hospitalares que se encontram, graças ao contexto pandêmico, sob escassez de recursos referidos ao processo de anestesia geral (AG).

\section{Metodologia}

Para cumprir com os requisitos da pesquisa e responder a pergunta norteadora: "Qual o papel da anestesia local e regional no contexto de saúde durante a pandemia de COVID-19?" dois revisores realizaram uma busca nos buscadores eletrônicos: Pubmed, Web of Science (todas as bases de dados da plataforma), Scopus, Embase e Cochrane Library. Contemplando as principais bases com o mais alto nível de evidência científica em saúde.

Para a busca de evidência foi utilizada a seguinte estratégiabaseada nos termos MeSH (Medical Subject Headings): ((SARS-CoV-2 OR COVID-19) AND (Anesthesia, Local OR Anesthetics, Local)). Tal estratégia foi repetida em todos os buscadores com exceção da plataforma Embase a qual possui descritores próprios sugeridos, logo os termos Emtree foram utilizados. Nenhum filtro foi utilizado, entretanto, cabe-se destacar que os achados datam a partir do ano de 2019 , uma vez que 
o vírus estudado ganhou destaque a partir dessa data. Todos os desenhos de estudo foram incluídos e não houve restrição quanto o idioma de publicação.

Os revisores sumarizaram os dados por meio de uma tabela de Excel com as informações: autor, ano, objetivo, área da medicina em foco e desfecho. Tal tabela foi utilizada exclusivamente para a descrição qualitativa dos dados apresentados (Oliveira et al., 2020).

\section{Resultados e Discussão}

Diante da realidade exposta e com base na metodologia empregada, o presente estudo visa elencar possíveis práticas que sustentem a troca da anestesia geral pelas demais, principalmente no contexto da pandemia, dessa forma, existe a necessidade de abordagem da temática de forma ampla de modo a sustentar o nível de evidência científica para o máximo de pacientes possíveis, permitindo a alocação de recursos necessários para a AG somente para casos estritamente necessários, dessa forma, o foco estudado transpassa a geração de aerossóis somente.

\subsection{Anestesia geral e regional no contexto da pandemia da COVID-19}

Frente a alta virulência do SARS-Cov-2 e a continuidade dos procedimentos de urgência e emergência, o setor de anestesiologia necessitou avaliar e reestruturar as práticas comuns de modo a combater a potencial exposição e transmissão do novo coronavírus no âmbito hospitalar e ambulatorial, preservando a saúde da equipe médica e a qualidade do atendimento prestado ao paciente (Gangakhedkar \& Chincholi, 2020).

Os anestesiologistas trabalham regularmente com procedimentos geradores de aerossóis, como a anestesia geral (AG), a qual apresenta alta capacidade de exposição e transmissão das partículas virais da COVID-19, colocando em risco os profissionais de saúde e demais pacientes hospitalizados independente da gravidade clínica da doença (Gangakhedkar \& Chincholi, 2020; Uppal et al., 2020). Uma recente revisão sistemática avaliou a transmissão de uma infecção respiratória aguda entre profissionais da saúde expostos a procedimentos geradores de aerossol e concluiu que as chances são aumentadas significativamente em 6,6 vezes aos expostos à prática (Herman et al., 2020).

Geralmente a AG apresenta a necessidade de administração medicamentosa como opioides, anti-inflamatórios e adjuvantes desde o transoperatório até o período seguinte a cirurgia para controlar a dor e estes, podem interagir negativamente com os protocolos medicamentosos atualmente utilizados no manejo da COVID-19 e somado as alterações fisiopatológicas da doença, causar graves efeitos adversos. Ademais, após um procedimento com AG é comum os pacientes apresentarem náuseas e vômitos, aumentando o risco de infecção dos profissionais de saúde e de desconforto ao paciente (Moreira et al., 2020).

Diante do exposto, estratégias que minimizem a aerolização e consequente a exposição e transmissão do SARS-CoV2, garantindo a segurança dos pacientes e profissionais da saúde, também reduzem o consumo de recursos e previnem efeitos adversos, cruciais na pandemia de coronavírus. Nesse contexto, diversos procedimentos eletivos, de urgência e emergência passaram por modificações no protocolo quanto a escolha da prática anestésica empregada, onde a anestesia regional (AR) ganhou destaque. Entende-se por AR a administração percutânea de anestésicos locais (AL) reduzindo a sensibilidade nervosa em uma parte especifica do corpo (Chin et al., 2021).

Um recente estudo conduzido por Cesur et al. (2020) com 126 médicos turcos mostrou que 42,6\% dos participantes relataram um aumento nas práticas de AR em suas aplicações anestésicas, dentre elas a anestesia neuroaxial, durante a pandemia da COVID-19, enquanto 57,4\% não relataram mudanças (Cesur et al., 2020). Já um estudo de coorte de casos de cirurgias de emergência e provisão de AR em um hospital no Reino Unido mostrou que em 34\% (26\% como técnica primária) dos procedimentos houve emprego da AR proeminentemente em cirúrgica geral, ginecológica e urológica (Wade et al., 2020).

A AR apresenta vantagens sobre a AG por preservar a função respiratória prevenindo complicações pós-operatórias e 
evitar a aerossolização e manipulação das vias aéreas, sendo considerada a técnica de primeira escolha em pacientes acometidos pela COVID-19 quando a condição sistêmica e o estádio de progressão clínica ou extensão da doença permitirem (Ashokka et al., 2020). Ainda, pode reduzir a morbidade perioperatória e fornecer analgesia prolongada (frequentemente 24 horas) sem sedação, diminuindo a administração e efeitos adversos das terapias farmacológicas para o manejo da dor pósoperatória, hemodinâmica estável, necessidade reduzida de outros anestésicos e oxigênio e rápida recuperação. Benefícios adicionais incluem redução de náuseas e vômitos e redução de disfunção cognitiva e alucinações (Ashokka et al., 2020; Cesur et al., 2020; Gangakhedkar \& Chincholi, 2020; Herman et al., 2020; Moll et al., 2021; Moreira et al., 2020; Ponde et al., 2020; Uppal et al., 2020).

Não há evidências científicas que a AR afeta negativamente a saúde dos pacientes acometidos pela COVID-19 (Hotta, 2021) e dada as vantagens acima listas da AR sobre a AG no manejo de pacientes infectados pelo novo coronavírus esta revisão narrativa visa explorar as considerações práticas para a aplicação de anestesias em paciente suspeitos ou com confirmação da doença, com foco nos procedimentos cirúrgicos realizados por especialidade e descritos a seguir.

\subsection{Mastologia}

O tratamento do câncer de mama é multidisciplinar e a pandemia de coronavírus exigiu o desenvolvimento de novas estratégias de mitigação para tal, de modo a fornecer cuidados apropriados e seguros aos pacientes acometidos durante o surto da COVID-19. De acordo com Sorbo et al. (2020) pacientes oncológicos são mais suscetíveis a infecção por COVID-19 e apresentam alto risco de evolução desfavorável frente a imunodeficiência provinda da condição clínica e tratamento farmacológico. Por esta razão, pacientes com câncer de mama e demais necessitam de um tratamento que equilibre o risco de progressão tumoral e de contaminação do coronavírus (Sorbo et al., 2020). Para isso, as adaptações perioperatórias incluíram a preferência por a AR (Cadili et al., 2021) para cirurgias emergenciais que apresentam alto risco de vida, classificadas em procedimentos de prioridade cirúrgica A e B (B1) (Holmes, 2020).

Cadili et al. (2020) avaliou o efeito da COVID-19 na quantidade e necessidade de adaptações a cirurgias de pacientes com câncer de mama na Columbia Britânica entre março a abril de 2019 e no mesmo período em 2020. Durante o período analisado, 93 de 162 (57\%) dos pacientes com câncer de mama receberam apenas AR em 2020, em comparação com 3 de 99 (3\%) em 2019 ( $\mathrm{p}=0,00001$ ), revelando um aumento da AR ao invés da AG em 2020 para evitar o procedimento de intubação durante a pandemia. Isso implicou em uma diferença estatisticamente significante nas altas no mesmo dia da cirurgia, sendo de 68\% em 2019 e $93 \%$ em 2020 (p =0,00001) e maior qualidade das cirurgias. Os autores concluem que a pandemia mudou o tratamento cirúrgico do câncer de mama para além da era pandêmica com a adoção de técnicas anestésicas mais seguras para os pacientes e profissionais da saúde (Cadili et al., 2021).

Por outro lado, Dias et al. (2020) relatou um caso clínico de mastectomia simples envolvendo uma paciente de 70 anos, diagnosticada com carcinoma ductal invasivo na mama esquerda. A paciente relatou ser portadora de cardiopatia durante a avaliação anestésica e, portanto, foi proposto o emprego da AR por esta reduzir a atividade do sistema nervoso simpático (SNS) e consequentemente, reduzir o débito e consumo de oxigênio cardíaco, com a combinação do bloqueio nervo peitoral (Pecs II), fascial pecto-intercostal (PIFB) e do nervo supraclavicular guiado por ultrassom. Vale ressaltar que o emprego de AR em cirurgias mamarias raramente são utilizadas como técnica primária dada a complexidade de inervação das mamas. Foi concluído que a escolha da anestesia foi uma opção válida para o procedimento, especialmente durante a pandemia de coronavírus, reduzindo a necessidade dos cuidados intensivos (Días et al., 2021).

Batt et al. (2020) avaliou se a dose máxima padronizada de lidocaína, um AL, forneceria analgesia eficaz no período perioperatório e pós-operatório quando diluída $(<1 \mathrm{mg} / \mathrm{mL})$, reduzindo a preocupação da toxicidade relativa comparando-a com um grupo controle. Foi observado aumento significativo da dor pós-operatória no grupo controle quando comparado com 
o grupo experimental. Portanto, os autores concluem que o AL diluído é capaz de fornecer uma abordagem alternativa eficaz e segura para procedimentos cirúrgicos de câncer de mama, evitando a AG durante a pandemia da COVID-19 (Batt et al., 2020).

\subsection{Ginecologia, obstetrícia e neonatos}

A pandemia de COVID-19 trouxe alguns questionamentos relevantes para as cirurgias ginecológicas. Os casos urgentes e emergenciais, como doenças malignas devem ser priorizados e se possível sob AR ou AL. Em relação aos procedimentos eletivos, as pacientes devem realizar o teste universal para coronavírus 40 horas antes das cirurgias e, em casos de testes positivos, a cirurgia deverá ser adiada. Quando aplicável, a laparoscopia é recomendada e durante procedimentos abertos e vaginais e a sucção pode ser usada para minimizar a propagação de gotículas e aerossol (Thomas et al., 2020).

Diferentemente dos procedimentos cirúrgicos eletivos, a obstetrícia não pode ser adiada ou cancelada e diante da pandemia em curso, o manejo das parturientes teve de ser reavaliado e readaptado para reduzir os riscos de disseminação do vírus. Neste contexto, a decisão sobre o tipo de parto e a prática anestésica permaneceu exclusivamente dependente da avaliação obstétrica (Ung \& Bonnet, 2020).

Ung \& Bonnet (2020) relataram uma proporção significativa de cesarianas de pacientes infectadas na China durante a doença COVID-19 e afirmam que esta escolha se baseia na prevenção da infecção vertical materno-fetal. Nestes casos, é lógico o emprego de AR, pois evita lesões por barotrauma e volutrauma, além das demais vantagens mencionadas anteriormente. No entanto, seu tempo médio de preparação e execução são relativamente maiores quando comparados com a AG, podendo implicar na necessidade do uso desta última em casos de emergência. Ademais a AG pode ser indicada nas pacientes que apresentam criticidade nas condições respiratórias, devido ao risco de desestabilização respiratória nos casos de falha na AR perimedular e sofrimento fetal agudo (Ung \& Bonnet, 2020; Varandas et al., 2021).

Batistaki et al. (2020) relataram o primeiro caso na Grécia do manuseio anestésico de uma paciente de 24 anos positivada para coronavírus, agendada para cesariana eletiva na quadragésima semana gestacional. Para o procedimento, a AR com equipamentos de proteção individual e coletivos completo para os profissionais de saúde envolvidos, associado a um planejamento minucioso foi realizada, alcançando um parto cesáreo seguro e sem intercorrências para parturientes e recémnascido (Batistaki et al., 2020).

Vale ressaltar que durante procedimentos neuroaxiais, os anestesiologistas devem se atentar sobre os riscos associados de meningite ou encefalite no contexto de viremia não tratada. Uma revisão bibliográfica identificou 14 casos admitidos entre janeiro e fevereiro de 2020 de parturientes positivadas para COVID-19 submetidas a procedimentos neuroaxiais, não sendo relatado sequelas neurológicas associadas. Destes, 13 (93\%) pacientes foram submetidas a cesariana sendo 6 (43\%) partos prematuros (<37 semanas de gestação) (Bauer et al., 2020). Segundo \& Bonnet (2020) não há evidências da suscetibilidade das parturientes à infecção viral, mas quando presente, a principal consequência é a prematuridade induzida e as complicações decorrentes. Ainda, 10 (71\%) pacientes apresentaram febre e 2, trombocitopenia. A febre decorrente da COVID-19 não é uma contraindicação para o empregado de uma AR peridural ou raquidiana, em contrapartida, é recomendado que os procedimentos sejam realizados em pacientes que apresentem ausência de trombocitopenia (Ung \& Bonnet, 2020).

Na série de casos publicada por Chen et al. (2020), foi avaliado o manejo e segurança da anestesia peridural ou AG para cesarianas em 17 parturientes com COVID-19 e seus recém-nascidos. Do total, 14 (82,4\%) parturientes foram submetidas a peridural e $12(70,6 \%)$ apresentaram hipotensão intraoperatória significativa. Em três parturientes foi utilizado a AG por emergência cirúrgica. Quanto aos recém-nascidos, três nasceram prematuramente, mas não houve relatos de mortes ou eventos graves relacionados ao parto, ainda, todos os testes neonatais de ácido nucléico SARS-CoV-2 foram negativos. Os autores concluem que ambas as anestesias foram empregadas com segurança para os partos cesáreos durante a pandemia de coronavírus, entretanto, a frequência de hipotensão nas parturientes sob anestesia peridural foi significativa (Chen et al., 2020). 
Bathia et al. (2020) em seu estudo transversal explorou se as taxas de AG para cesariana mudaram durante a pandemia da COVID-19 em 6 maternidades inglesas durante abril a julho de 2020 quando comparado a um período semelhante de 2019. Foi observado uma redução significativa no emprego de AG de 7,7\% para 3,7\% (p <0,0001), com a taxa de utilização da AR aumentando para mais de 96\%, ainda as taxas de conversão da AR para AG reduziram de 1,7\% para 0,8\% (p =0,01) e a taxa geral de partos cesáreos aumentou de 28,3\% para 29,7\% (p =0,052) (Bhatia et al., 2021).

Zhong et al. (2020) avaliaram retrospectivamente 49 pacientes com COVID-19 confirmados para cesariana (45 91\%) ou cirurgia de membro inferior (4 - 9\%) submetidas a raquianestesia (AS) (ropivacaína 0,75\%) em um hospital da China. Observou-se que a AS não foi associada no intraoperatório a comprometimentos cardiorrespiratórios e os pacientes não desenvolveram pneumonia grave posteriormente. Os anestesistas realizaram os procedimentos utilizando equipamentos de proteção individual (EPI) nível 1 ou 3 e dos 44 profissionais, 5 (11,4\%) se infectaram com COVID-19. Concluiu-se que a AS foi aplicada com segurança e os EPIs nível 3 podem reduzir a taxa de infecção de exposição e transmissão do coronavírus (Zhong et al., 2020).

\subsection{Urologia}

Durante a pandemia da COVID-19 as práticas padrões para o gerenciamento dos cálculos urinários passaram por reavaliação e até o momento, não há diretrizes claras para o manejo destes (Fakhr Yasseri \& Aghamir, 2020; Gökce et al., 2020).

Usualmente, o tratamento de cálculos urinários pode ser divido em dois grupos, onde o primeiro não compreende as intervenções urológicas e são indicadas modificações dietéticas e do estilo de vida e o segundo as compreende e pode ser subdividido em emergenciais e não emergenciais. O subgrupo emergencial inclui pacientes com cálculos urinários e uremia, sepse, anúria ou dor refratária e vômitos concomitantes, sendo a opção intervencionista durante a pandemia de coronavírus a inserção de tubo de nefrostomia percutânea (NCP) e stents uretrais sob AL ou AR (raquianestesia ou peridural). Apesar de ser um método eficaz e seguro foi relatado uma maior taxa de falha na inserção de stent em pacientes sob AL quando comparados com pacientes sob AG, não sendo estatisticamente significante (8,7\% vs 1,3\% - p = 0,07) (Fakhr Yasseri \& Aghamir, 2020; Tonyali et al., 2020).

Já o subgrupo não emergência inclui pacientes com cálculos renais e uretrais assintomáticos e sem complicações o qual as intervenções urológicas são indicadas, porém, durante o surto da COVID-19 é sugerido tratamento conservador por três meses e após, uma reavaliação da condição clínica do paciente (Fakhr Yasseri \& Aghamir, 2020; Tonyali et al., 2020).

Vale ressaltar que neste momento, apenas intervenções emergenciais devem ser realizadas visto que o não tratamento destas pode acarretar em complicações e efeitos negativos sob a função renal a longo prazo (Fakhr Yasseri \& Aghamir, 2020; Gökce et al., 2020).

Gökce et al., (2020) avaliaram em seu estudo as práticas atualizadas relacionados à avaliação pré-operatória e a anestesia para intervenções envolvendo cálculos durante a pandemia da COVID-19 em 11 centros de 5 países diferentes de forma retrospectiva. Quanto as condições clínicas, o cálculo renal foi o mais prevalente (38,5\%), seguido por cálculo uretral $(23,9 \%)$, múltiplos cálculos - renais e uretrais $(21,9 \%)$ e por fim, pielonefrite obstrutiva $(9,8 \%)$, onde o procedimento mais comum realizado durante o estudo foi a extração de cálculo ureteroscópico $(34,5 \%)$. Quanto as práticas de avaliação préanestésica foram observadas com maior frequência a tomografia computadorizada (TC) de tórax (268 - 56,7\%), seguido da PRC do swab nasofaríngeo $(31$ - 6,6) e o teste rápido de antígeno foi realizado em apenas um paciente. Em relação a prática anestésica, a AG intratraqueal ou máscara laríngea foi aplicada em 337 (71,2\%) pacientes, raquinestesia/peridural em 76 $(16,1 \%)$ e AL em $52(11 \%)$ pacientes. Por fim, concluem-se a taxa de testes pré-operatórios, procedimentos de emergência, abordagens conservadoras e AR/AL aumentaram após 21 dias (valor de corte) e, apesar da AG acontecer em maior frequência, 
a AR pode fornecer uma anestesia bem-sucedida e útil na proteção à exposição da equipe médica, mas levantam a hipótese que o SARS-CoV-2 pode envolver o sistema nervoso central (SNC) e, portanto, a transmissão também pode ocorrer durante a AR, a qual não oferece uma proteção completa (Gökce et al., 2020).

Outra complicação urológica que acomete grande parcela da população mundial é o câncer de bexiga, onde procedimentos cirúrgicos são fundamentais para diagnóstico e tratamento destes na forma de ressecção transuretral de tumor de bexiga (TURBT). Sendo mais prevalente em pacientes após os 60 anos (média de 73 anos) e considerando as comorbidades relacionadas a idade, a AS é a escolha para este grupo de pacientes, visto que a AG pode acarretar o aumento do risco de complicações cardiorrespiratórias (Krishan et al., 2021).

Krishan et al., (2020) investigaram através de uma meta-análise se a AS com bloqueio do nervo obturador (ONB) é eficaz para TURBT durante a pandemia de coronavírus, comparando os resultados de TURBT com AS e AS associado ao ONB. Os resultados mostram que o emprego de AS associado ao ONB apresentou uma incidência significativamente menor de reflexo obturador, perfuração da bexiga, TURBT incompleta e recorrência do tumor em doze meses quando comparado com o emprego de AS isoladamente, indicando uma ressecção de maior qualidade do tumor. Ainda, não foi encontrado na literatura evidências da toxicidade do AL utilizado. Diante disto, os autores concluem que a AS apresenta vantagens durante a pandemia da COVID-19 e deve ser preferida sempre que possível (Krishan et al., 2021).

\subsection{Oftalmologia}

Segundo Ahmad et al. (2020) durante a pandemia de coronavírus a oftalmologia apresentou um papel particular frente a ausência de evidências sobre os cuidados perioperatórios de cirurgia oftálmica em pacientes acometidos pela COVID-19 (Ahmad et al., 2020).

Os profissionais oftalmologistas e anestesiologistas necessitam realizar bloqueios regionais oftálmicos para procedimentos cirúrgicos em pacientes confirmados ou não com COVID-19, entrando em contato direto com o tecido conjuntivo ocular e lágrimas destes. Diante disto, e levando em consideração que podem ocorrer a comunicação verbal, tosse ou espirros inesperados durante o procedimento, disseminando o SARS-CoV-2, estes profissionais estão expostos e podem ser potencialmente infectados pelo coronavírus. Neste contexto e durante a prática da AR recomenda-se a sedação consciente oral ou intravenosa, prevenindo os reflexos nervosos e eventuais imprevistos que possam comprometer a saúde dos profissionais e também, dos demais pacientes. Por outro lado, a sedação profunda deve ser evitada por conta das possíveis complicações pulmonares causadas pela doença (Ahmad et al., 2020).

Alguns procedimentos de diagnósticos e cirúrgicos oftalmológicos necessitam do emprego de AG para as cirurgias oftálmicas, em especial em pacientes pediátricos, sob intubação endotraqual, representando um risco potencial de exposição e transmissão do vírus aos profissionais de saúde (Ahmad et al., 2020).

Rajput, et al. (2020) avaliou prospectivamente o perfil de segurança da cetamina, fármaco antagonista não competitivo do receptor NMDA, combinada a AR em cirurgias oculares pediátricas durante a pandemia de COVID-19. 55 crianças (62 olhos operados) participaram da pesquisa, onde a laceração da pálpebra foi o procedimento cirúrgico mais comum $(32,7 \%)$. Foram administrados midazolam oral $(0,25-0,50 \mathrm{mg} / \mathrm{kg})$ ou cetamina intramuscular (IM) (7-10 mg / kg), ondensetrona $(0,1 \mathrm{mg} / \mathrm{kg})$ e atropina $(0,02 \mathrm{mg} / \mathrm{kg})$. A anestesia foi obtida com cetamina intravenosa (IV) (4-5 mg / kg) e AL (bloqueio peribulbar ou infiltração local). Não houve efeitos colaterais graves, nenhum paciente necessitou de intubação e nenhuma ressuscitação foi necessária. Quatro crianças queixaram-se de náuseas e duas necessitaram de dose adicional de ondansetrona IV devido a vômitos no pós-operatório. Conclui-se que a combinação de cetamina e AR é uma alternativa segura e eficaz para administrar anestesia em crianças durante cirurgias oculares (Rajput et al., 2021). 


\subsection{Ortopedia}

Os ortopedistas em todas as especialidades, em resposta as mudanças das prioridades clínicas e os recursos das cirurgias eletivas, de urgência e emergência, consequentes da pandemia da COVID-19, demonstraram grande capacidade para o desenvolvimento e disseminação de protocolos e diretrizes, utilizando novas abordagens cuidados aos pacientes cirúrgicos (Turcotte et al., 2020).

Um estudo comparativo sobre trauma e eficiência cirúrgica ortopédica realizadas em Cariff e no Conselho de Saúde da Universidade Vale coletou dados de 222 pacientes submetidos a procedimentos ortopédicos de emergência em maio de 2019 e 161 pacientes em maio de 2020 quanto ao tempo de preparação anestésica, tempo de anestesia, tempo de preparação cirúrgica, tempo cirúrgico, tempo de transferência para recuperação e o tempo de resposta. Foi observado um aumento estatisticamente significativo em todos os tempos analisados, exceto para o tempo de anestesia. Quanto as práticas anestésicas, em 2019 a AG combinada com AR foi utilizada em 178 (80,18\%) pacientes, AL em 10 (4,5\%) e AR em 34 (15,3\%) pacientes, respectivamente, enquanto em 2020, a AG combinada com AR foi utilizada em $131(81,4 \%)$ pacientes, AL em $15(9,3 \%)$ e AR em 15 (9,3\%) pacientes, respectivamente. Observa-se que em 2020 a quantidade de procedimentos cirúrgicos ortopédicos foi menor quando comparada com 2019 por conta da priorização dos serviços em decorrência da COVID-19, mas mesmo assim, a frequência do emprego da AR aumentou no período, sugerindo sua eficiência para as cirurgias ortopédicas (Mercer et al., 2020).

Em torno de $10 \%$ das fraturas proximais do úmero ocorrem em pacientes idosos, requerendo cirurgia de emergência e um grande desafio para os anestesistas. Após o surto de COVID-19 no norte da Itália, o bloqueio interescalênico (IS) está sendo frequentemente associada ao bloqueio supraclavicular (SC) (bloqueio IS-SC), sempre que possível, para que as cirurgias das fraturas proximais do úmero sejam realizadas completamente por AR em pacientes positivados ou suspeitos para coronavírus. Para isto, uma mistura de 1:1 de mepivacaína $2 \%$ e levobupivacaína 0,5\% é utilizada para o bloqueio IS-SC. Acredita-se que esta prática represente uma alternativa válida a AG na condição em questão, especialmente nos pacientes que apresentam a infecção respiratória viral em curso e por reduzir a exposição aos profissionais de saúde ao contato viral (Tognù et al., 2021).

O procedimento com AL e com paciente acordado e sem torniquete representado pela sigla WALANT (do inglês Wide Awake Local Anesthetic No Tourniquet procedure) apresenta uma abordagem anestésica alternativa e de destaque a prática de AG e AR que constituem a base para procedimentos cirúrgicos ortopédicos. O WALANT, consiste na injeção préincisão de um AL no local da cirurgia, fornecendo analgesia e vasoconstrição mitigando o sangramento no campo operatório em vez de um torniquete e não requer a presença de um anestesista para tal e tem sido utilizado com sucesso nas cirurgias de mão, pé e tornozelo, ulna do eixo médio e fraturas do rádio distal (Turcotte et al., 2021).

Turcotte et al. (2020) relataram uma série de casos com a implementação do WALANT em vez da AG, sempre que possível, em 16 procedimentos ortopédicos durante a pandemia da COVID-19, tendo como desfecho primário a falha de WALANT, definida para conversão intraoperatória para AG. Durante o estudo, nenhuma falha do WALANT que exigisse a conversão para AG foi registrada e apenas um (6\%) paciente necessitou de medicamentos para analgesia pós-operatória. Concluíram que o WALANT foi usado com segurança e eficácia nos casos avaliados nas subespecialidades e que a técnica pode ser empregada para tratar uma gama de patologias ortopédicas, podendo ser incorporadas na rotina dos procedimentos padrões (Turcotte et al., 2021). Ainda, a técnica WALANT foi empregada com sucesso para procedimentos cirúrgicos e tratamento dos tendões flexores (Ayhan et al., 2021).

Sadr et al. (2020) expões que antes da pandemia em um hospital do Reino Unido, 50\% dos traumas tratados foram conduzidos sob AG, $33 \%$ sob AL e 17\% sob bloqueio de nervos periféricos ou branquiais e durante este momento, realizaram com sucesso 95\% cirurgias utilizando o WALANT, demonstrando um aumento desta técnica como primeira escolha para os 
procedimentos ortopédicos (Sadr et al., 2020). Já Saha et al. (2020) resumiram retrospectivamente o manejo de 102 traumas de mão e como as modificações nas práticas otimizaram os cuidados aos pacientes em um Departamento de Cirurgia Plástica na India entre março a maio de 2020. Cinco pacientes eram COVID-19 positivos. Do total, 10 pacientes receberam AG (6 devido a falhas da AR), 75 (73,5\%) e 17 (16,7\%) pacientes foram submetidos a procedimentos sob AL/WALANT e sob bloqueio do plexo braquial, respectivamente. Os autores frisam a necessidade da flexibilidade e dinamicidade nas abordagens ortopédicas, utilizando sempre que possível a WALANT para traumas e suspeita de infecção por COVID-19 (Saha et al., 2021).

Usualmente as cirurgias da coluna são feitas sob AG, mas atualmente há evidências de procedimentos bem-sucedidos sob AS. Sarkar et al. (2020) analisaram retrospectivamente os dados de 131 pacientes submetidos a cirurgias de fusão instrumentada da coluna lombar (estenose lombar e espondilolistese degenerativa ou lítica) sob AS e 108 sob AG quanto a segurança, vantagens e desvantagens. Os resultados mostraram redução significativa de perda de sangue ( $p<0,05)$, menor tempo de permanência no centro cirúrgico, melhor analgesia pós operatória e menor incidência de náuseas e vômito nos pacientes sob AS $(8,4 \%)$ do que sob GA $(29,6 \%)$. Nenhuma complicação relacionada a posição prona na AS foi relatada, mas uma paralisia transitória do plexo branquial e dor no ombro pós-operação foi relatada nos pacientes sob AG. Os autores concluem que a AS é uma alternativa segura a AG para cirurgias de fusão instrumentada da coluna lombar frente as vantagens descritas acima (Sarkar et al., 2021).

Apesar da fratura do osso nasal ser uma apresentação comum aos cirurgiões otorrinolaringologistas, o tema será tratado neste tópico. Estas fraturas correspondem a 39\% de todas as fraturas do osso facial, especialmente por sua proeminência na face (Bastianpillai et al., 2020). Em um departamento de otorrinolaringologia no Reino Unido, antes da pandemia de coronavírus, as cirurgias de fraturas do osso facial eram realizadas sob AG, porém, durante este período, houve uma mudança para a realização dos procedimentos os quais passaram a ser realizados sob AL no ambulatório, para evitar a indisponibilidade dos centros cirúrgicos e oferecer aos pacientes e profissionais um serviço seguro. Para avaliar a qualidade da transição destes serviços, os prontuários dos pacientes entre janeiro a agosto de 2020 foram analisados retrospectivamente e estes, acompanhados por um mês após a cirurgia. No total, 21 e 27 procedimentos foram realizados sob AG e AR, respectivamente. Os pacientes relataram satisfação respiratória de 2,88 $\pm 0,24$ para 4,06 $\pm 0,23$ (p <0,05) sob AG e de 2,86 \pm 0,22 para 3,77 $\pm 0,27(\mathrm{p}<0,05)$ sob AR e para os escores estéticos $(\mathrm{p}<0,05)$ em ambos os grupos. Não foi observado diferença estatisticamente significativa entre AL e AG quanto aos resultados pós-operatórios, embora houve uma tendência maior para o AG frente ao impacto pela taxa de deformidade cartilaginosas no grupo AL. Foi concluído ao final, que a AL é uma alternativa segura, barata e satisfatória para a realização de procedimentos cirúrgicos de fraturas ósseas nasais em relação a AG (Bastianpillai et al., 2020).

\subsection{Neurologia}

Os procedimentos cirúrgicos neurológicos não se isentaram do impacto da pandemia de coronavírus e levando em consideração a proximidade do campo operatório ao trato respiratório superior, neurocirurgias em pacientes com COVID-19 é desafiador (Okunlola, 2021).

Okunlola (2020) relatou os desafios e resultados de uma craniotomia para retirada de um glioma parieto-occipital esquerdo em uma paciente idosa positiva para COVID-19 e hipertensa em um hospital de ensino nigeriano. O procedimento foi realizado sob AR não tendo a necessidade de conversão para AG intraoperatório. Ademais, a paciente teve melhora imediata da função motora no pós-operatório e ganho motor confirmado após 30 dias do procedimento. O autor concluiu que a craniotomia acordada em pacientes infectados pelo SARS-CoV-2 é alcançável (Okunlola, 2021).

A trombectomia mecânica (TM), considerada como o tratamento padrão do acidente vascular cerebral (AVC) isquêmico agudo gera grande debate quanto ao melhor método anestésico para o procedimento, sendo a AG, AL e sedação 
consciente (SC) considerados. A literatura apresenta dados comparativos entre AG e SC inconsistentes e não há evidências sobre o emprego da AL na TM, portanto, não é conhecido sua real eficácia (Anadani et al., 2021).

Anadini et al. (2020) compararam a AL com SC em 874 pacientes (636 no grupo SC e 238 no grupo AL) utilizando registros prospectivos no tratamento endovascular em AVC isquêmico. A análise combinada de escore de propensão, que incluiu 222 pacientes em ambos os grupos, o AL apresentou uma taxa inferior de resultados funcionais favoráveis (escala de Rankin modificada [mRS] 0-2; 40\% vs. 52\%, risco relativo compatível = 0,76 [intervalo de confiança de 95\%, 0,60-0,97]) e reperfusão bem-sucedida $(76,6 \%$ vs. $87,1 \%$; risco relativo correspondente $=0,88$ [intervalo de confiança de 95\%, 0,79-0,98]) em 90 dias, sugerindo que a SC permitiu maior taxa de sucesso e melhores resultados na TM (Anadani et al., 2021). Em contrates com estes achados, Goldhoorn et al. (2020) relatou a associação entre AG e SC com ruins resultados funcionais em 90 dias quando comparados com AL. Embora o resultado neurológico sob AL tenha sido semelhante ao estudo anterior (mRS 0-2:40\%), sob SC foi consideravelmente baixo (mRS 0-2 :25\%) (Goldhoorn et al., 2020).

\subsection{Cardiologia e vascular}

Os pacientes acometidos pela COVID-19 podem desenvolver complicações cardíacas graves como miocardite, insuficiência cardíaca e arritmias. Dada esta informação, e levando em consideração o adiamento das consultas e procedimentos cirúrgicos eletivos, é de suma importância que ocorra um diagnóstico, isolamento e manejo clínico intensivo e imediato, em especial nos pacientes já cardiopatas (Balgaith et al., 2020; Dendramis \& Brugada, 2020).

Neste contexto, a doença arritmogênica conhecida como síndrome de Brugada $(\mathrm{BrS})$ é uma das principais causas de morte cardíaca em pacientes com menos de 40 anos. Para decidir qual a técnica anestésica deve ser utilizada em pacientes com Brs, deve-se levar em consideração os medicamentos e outros fatores que possuem capacidade de induzir arritmias. A AG por via inalatória ou associada a opiáceos e agentes de indução como propofol, tiopental e etomidato pode ser realizada com segurança nestes pacientes. Entretanto, observou-se que o propofol em bolus ou infusão pode elevar significativamente o segmento ST em pacientes com BrS e síndrome de infusão de propofol, caracterizada por acidose metabólica, disfunção cardíaca, rabdomiólise, hipertrigliceridemia ou insuficiência renal. Já a AR e o bloqueio neuroaxial devem ser realizados com cautela visto que os AL são antiarrítmicos e bloqueiam os canais de sódio voltagem dependente, especialmente a ropivacaína, bupivacaína e levobupivacaína. Por conta disto, deve-se minimizar as doses dos AL e monitorar o paciente de forma intensiva e quando possível, optar por outra técnica anestésica (Dendramis \& Brugada, 2020).

Sobre a especialidade vascular, durante a pandemia de coronavírus as complicações bem como os profissionais vasculares tiveram diferentes desfechos (Shih et al., 2020) como os descritos a seguir.

A esteanose aórtica é um estreitamento da abertura da válvula aórtica que obstrui o fluxo sanguíneo do ventrículo esquerdo para a aorta e requer intervenção cirúrgica rápida para inserção do implante de válvula aórtica transcateter (TAVI) e atinge principalmente os pacientes idosos (Balgaith et al., 2020). Durante a pandemia em curso, de acordo com Balghith et al. (2020) a AG para a inserção de TAVI é seguro e estável, porém, é mais viável o emprego da AL com SC principalmente por ser menos invasivo, agilizar o procedimento cirúrgico e de recuperação e reduzir o tempo de internação hospitalar, podendo ser considerada a melhor técnica de anestesia durante essa crise, quando aplicável. No entanto, não se deve descartar a necessidade não planejada da conversão para AG intraoperatório (Balgaith et al., 2020).

Um relato de caso de Rossi et al. (2020) contou sobre o reparo endovascular da aorta torácica (TEVAR) em uma paciente de 64 anos SARS-CoV-2 positiva. A TC de tórax realizada após a chegada da paciente na emergência revelou dissecção aguda da aorta tipo B (ATBAD) e envolvimento pulmonar bilateral, compatível com pneumonia intersticial. O procedimento cirúrgico de TEVAR foi realizado sob AL sem intercorrências cirurgias e respiratórias e as dores no peito e na costa desapareceram após os primeiros dias após o procedimento. Rossi et al. (2020) concluíram que o procedimento com AL 
em pacientes infectados parece ser razoavelmente aplicável para TEVAR (Rossi et al., 2020).

Verikokos et al. (2020) relatam que o reparo endovascular de aneurisma (EVAR) pode ser realizado sob AL com um resultado bem-sucedido acompanhado de uma rápida recuperação para pacientes com ruptura de aneurismas de aorta abdominal (rAAAs), pois reduz os riscos perioperatórios e o tempo de internação pós-operatório. Um paciente de 78 anos foi submetido a EVAR na China com AL sem nenhuma intercorrência, com mobilização total e alimentação por via oral desde o primeiro dia de pós-operatório e no segundo, recebeu alta. O autor ainda cita que em uma recente análise, pacientes com rAAAs submetidos a EVAR com AL comparados com EVAR sob AG apresentaram redução do tempo intraoperatório e de internação, número de transfusões sanguíneas e complicações pulmonares e conclui que usar EVAR com AL parece ser a melhor solução no manejo dos pacientes com rAAAs, para segurança dos profissionais e economia dos recursos de saúde (Verikokos et al., 2020). Shih et al. (2020) também relataram situação semelhante, onde um paciente com COVID-19 e diagnosticado com rAAA de $5,8 \mathrm{~cm}$ com hematoma adjacente ao pâncreas foi submetido ao EVAR com AL, o procedimento teve bons resultados em curto prazo (Shih et al., 2020).

Vale ressaltar que alguns pacientes acometidos pela COVID-19 apresentam em algum grau coagulopatias sanguíneas ( $\geq 2$ vezes acima do normal) que se correlacionam com a criticidade da doença e influenciam nos procedimentos cirúrgicos, merecendo destaque, além do prolongamento do tempo de protombina (1-3 segundos acima do normal) e diminuição de fibrinogênio $(<2 \mathrm{~g} / \mathrm{L})$. Por isso, exames laboratoriais pré-cirurgia são de extrema importância e as precauções padrão da AR são recomendadas, em especial, interromper a anticoagulação em pacientes submetidos a terapias farmacológicas (Mendes et al., 2021).

\subsection{Cirurgias toracoabdominais}

Atualmente, grande parte das cirurgias abdominais são realizadas com técnicas minimamente invasivas (TMI), como cirurgia robótica e laparotomia sob AG. Na pandemia da COVID-19 a funcionalidade e segurança desta prática foi questionada (Romanzi et al., 2020; Romanzi et al., 2021)

Romanzi et al. (2021) relataram uma treze casos cirúrgicos inadiáveis sob AR (raquianestesia, peridural ou raquianestesia combinada) em pacientes idosos (média de 80 anos), os quais a exposição a AG e fatores relacionados poderiam aumentar as chances de efeitos adversos e morbimortalidade. A intensidade da dor foi monitorada no intra e pós-operatório. Apenas em um caso houve a necessidade de conversão para AG e não ocorreram complicações graves perioperatórias e nem necessidade de suporte intensivo pós-operatório. Foi concluído a viabilidade da laparotomia sob AR para cirurgias planejadas, frente a riscos de infecção e a pacientes com comprometimento cardiorespiratório, embora a AG seja frequentemente mais utilizada (Romanzi et al., 2021).

Em 2019 oito pacientes com idade média de 88 anos necessitaram de cirurgias abdominais devido a emergências gastrointestinais (GI) ou doenças oncológicas após terapia neoadjuvante. Os mesmos apresentavam doenças cardiorrespiratórias e múltiplas comorbidades, sendo considerados de alto risco (ASA >3) e, portanto, sugestivos a não tolerar AG. As cirurgias foram realizadas sob anestesia locorregional. A intensidade da dor intra e pós-operatória foi monitorada e regularmente avaliada por meio do uso da escala de classificação numérica (NRS). Nenhum paciente necessitou de intubação e suporte de terapia intensiva pós operatório, ademais, a dor intra-operatória e pós-operatória foram bem controladas, não sendo observadas complicações. Foi possível concluir que realizar grandes cirurgias sob AL é eficaz e segura, até mesmo em pacientes idosos e frágeis especialmente durante a COVID-19 (Romanzi et al., 2020).

\section{Conclusão}

A pandemia da COVID-19 trouxe diversos questionamentos e mudanças para a anestesiologia de procedimentos eletivos, 
urgentes e emergenciais, onde o planejamento e a comunicação multidisciplinar entre a equipe médica são cruciais para a escolha da técnica anestésica. Neste contexto, a AR ganhou destaque em pacientes com suspeita ou confirmação de COVID19, como uma opção viável, eficaz e segura a AG em diversas especialidades e procedimentos médicos cirúrgicos, garantindo a segurança dos pacientes e profissionais de saúde.

Dessa forma, estudos clínicos futuros podem utilizar-se dessa estrutura para continuar a executar procedimentos utilizando a técnica anestésica regional como alternativa a anestesia geral. Tal fator deve-se tanto a economia de recursos hospitalares, como a manutenção de um pós-operatório com uma menor dosagem de medicamentos analgésicos e anti-inflamatórios. subsidiando, assim, uma alternativa terapêutica possível para escolha da equipe médica em cenários adversos a anestesia geral.

\section{Referências}

Ahmad, N., Zahoor, A., Ahmad, A., \& El Dawlatly, A. (2020). Anesthesia management of ophthalmic surgery for patient with suspected/confirmed COVID19 -Saudi Anesthesia Society guidelines-. Saudi Journal of Anaesthesia, 14(3), 355. https://doi.org/10.4103/sja.SJA_260_20

Anadani, M., Audibert, G., \& Gory, B. (2021). Conscious Sedation versus Local Anesthesia During Thrombectomy for Acute Ischemic Stroke, Do We Have a Winner? World Neurosurgery, 146, 383-384. https://doi.org/10.1016/j.wneu.2020.12.001

Ashokka, B., Chakraborty, A., Subramanian, B. J., Karmakar, M. K., \& Chan, V. (2020). Reconfiguring the scope and practice of regional anesthesia in a pandemic: the COVID-19 perspective. Regional Anesthesia \& Pain Medicine, 45(7), 536-543. https://doi.org/10.1136/rapm-2020-101541

Association of Anesthetists, Royal College of Anesthetists, 2020. (2020). Guidance on potential changes to anaesthetic drug usage and administration during pandemic emergency pressures. https://icmanaesthesiacovid-19.org/drug-demand-supply-anaesthetic-drug-usage-and-administration

Ayhan, E., Tuna, Z., \& Oksuz, C. (2021). Getting Better Results in Flexor Tendon Surgery and Therapy. Plastic and Reconstructive Surgery - Global Open, 9(2), e3432. https://doi.org/10.1097/GOX.0000000000003432

Balgaith, M., Arifi, A., Ahmed, D., \& Metwally, A. M. (2020). The Impact of COVID-19 Pandemic on the Hospital Management of TAVI Patients: TAVI team Thoughts and Recommendation. Journal of the Saudi Heart Association, 32(5), 11-15. https://doi.org/10.37616/2212-5043.1030

Bastianpillai, J., Khan, S., Acharya, V., Tanna, R., \& Pal, S. (2020). How COVID-19 Changed Our Management of Nasal Bone Fractures and Its Impact on Patient Outcomes-A Retrospective Study. Ear, Nose \& Throat Journal, 014556132098143. https://doi.org/10.1177/0145561320981439

Batistaki, C., Galarioti, V., Vasiliadou, S., Soulioti, E., Kostopanagiotou, G., \& Matsota, P. (2020). Lessons learned from first case of Cesarean delivery in a COVID-19 positive parturient in Greek region. Journal of Anaesthesiology Clinical Pharmacology, 36(5), 121. https://doi.org/10.4103/joacp.JOACP_157_20

Batt, J., Cook, N., Nadeem, M., \& Sahu, A. (2020). Dilutional local anaesthetic techniques in oncoplastic breast surgery and potential benefits during the COVID-19 pandemic and beyond. Journal of Perioperative Practice, 30(9), 277-282. https://doi.org/10.1177/1750458920944080

Bauer, M. E., Chiware, R., \& Pancaro, C. (2020). Neuraxial Procedures in COVID-19-Positive Parturients: A Review of Current Reports. Anesthesia \& Analgesia, 131(1), e22-e24. https://doi.org/10.1213/ANE.0000000000004831

Bhatia, K., Columb, M., Bewlay, A., Eccles, J., Hulgur, M., Jayan, N., Lie, J., Verma, D., \& Parikh, R. (2021). The effect of COVID-19 on general anaesthesia rates for caesarean section. A cross-sectional analysis of six hospitals in the north-west of England. Anaesthesia, 76(3), 312-319. https://doi.org/10.1111/anae.15313

Cadili, L., DeGirolamo, K., McKevitt, E., Brown, C. J., Prabhakar, C., Pao, J.-S., Dingee, C., Bazzarelli, A., \& Warburton, R. (2021). COVID-19 and breast cancer at a Regional Breast Centre: our flexible approach during the pandemic. Breast Cancer Research and Treatment, 186(2), 519-525. https://doi.org/10.1007/s10549-020-06008-3

Cesur, S., Aksu, C., \& Kuş, A. (2020). Regional Anesthesia Practices in Turkey During the COVID-19 Pandemic. Cureus, 12 (8), e10135. https://doi.org/10.7759/cureus.10135

Chen, R., Zhang, Y., Huang, L., Cheng, B., Xia, Z., \& Meng, Q. (2020). Safety and efficacy of different anesthetic regimens for parturients with COVID-19 undergoing Cesarean delivery: a case series of 17 patients. Canadian Journal of Anesthesia/Journal Canadien d'anesthésie, 67(6), 655-663. https://doi.org/10.1007/s12630-020-01630-7

Chin, K. J., Mariano, E. R., \& El-Boghdadly, K. (2021). Advancing towards the next frontier in regional anaesthesia. Anaesthesia, 76(S1), 3-7. https://doi.org/10.1111/anae.15321

Coulthard, P. (2020). Dentistry and coronavirus (COVID-19) - moral decision-making. British Dental Journal, 228(7), 503-505. https://doi.org/10.1038/s41415-020-1482-1

Dendramis, G., \& Brugada, P. (2020). Intensive care and anesthetic management of patients with Brugada syndrome and COVID-19 infection. Pacing and Clinical Electrophysiology, 43(10), 1184-1189. https://doi.org/10.1111/pace.14044

Días, R., Mendes, Â. B., Lages, N., \& Machado, H. (2021). Bloqueos del plano fascial ecoguiados, como única técnica anestésica para mastectomía total en la era de la COVID-19: caso clínico. Revista Española de Anestesiología y Reanimación. https://doi.org/10.1016/j.redar.2020.09.010

Fakhr Yasseri, A., \& Aghamir, S. M. K. (2020). Urinary stone management during the COVID-19 pandemic: a suggested approach and review of literature. Therapeutic Advances in Urology, 12, 175628722093951. https://doi.org/10.1177/1756287220939513 
Gangakhedkar, G., \& Chincholi, I. (2020). Re-inventing anesthesia in times of Covid-19. Journal of Anaesthesiology Clinical Pharmacology, $36(5)$, 48. https://doi.org/10.4103/joacp.JOACP_189_20

Gökce, M. İ., Yin, S., Sönmez, M. G., Eryildirim, B., Kallidonis, P., Petkova, K., Guven, S., Kiremit, M. C., de Lorenzis, E., Tefik, T., Villa, L., Zeng, G., \& Sarica, K. (2020). How does the COVID-19 pandemic affect the preoperative evaluation and anesthesia applied for urinary stones? EULIS eCORE-IAU multicenter collaborative cohort study. Urolithiasis, 48(4), 345-351. https://doi.org/10.1007/s00240-020-01193-8

Herman, J. A., Urits, I., Kaye, A. D., Urman, R. D., \& Viswanath, O. (2020). COVID-19: Recommendations for regional anesthesia. Journal of Clinical Anesthesia, 65, 109885. https://doi.org/10.1016/j.jclinane.2020.109885

Holmes, D. R. (2020). Breast cancer care during a pandemic: an opportune time for cryoablation? Breast Cancer Research and Treatment, 182(3), 515-521. https://doi.org/10.1007/s10549-020-05724-0

Hotta, K. (2021). Regional anesthesia in the time of COVID-19: a minireview. Journal of Anesthesia, 35(3), 341-344. https://doi.org/10.1007/s00540-02002834-3

Krishan, A., Bruce, A., Khashaba, S., Abouelela, M., \& Ehsanullah, S. A. (2021). Safety and Efficacy of Transurethral Resection of Bladder Tumor Comparing Spinal Anesthesia with Spinal Anesthesia with an Obturator Nerve Block: A Systematic Review and Meta-analysis. Journal of Endourology, 35(3), 249-258. https://doi.org/10.1089/end.2020.1054

Mendes, A. B., Penedos, C., Vaz Rodrigues, L., Varandas, J. S., Lages, N., \& Machado, H. (2021). Coagulation concerns in patients with COVID-19 proposed for regional anesthesia. Regional Anesthesia \& Pain Medicine, 46(5), 457.1-457. https://doi.org/10.1136/rapm-2020-101676

Mercer, S. T., Agarwal, R., Dayananda, K. S. S., Yasin, T., \& Trickett, R. W. (2020). A comparative study looking at trauma and orthopaedic operating efficiency in the COVID-19 era. Perioperative Care and Operating Room Management, 21, 100142. https://doi.org/10.1016/j.pcorm.2020.100142

Moll, V., Mariano, E. R., Kitzman, J. M., O’Reilly-Shah, V. N., \& Jabaley, C. S. (2021). Regional anesthesia educational material utilization varies by World Bank income category: A mobile health application data study. PLOS ONE, 16(2), e0244860. https://doi.org/10.1371/journal.pone.0244860

Moreira, R., Andrade, L. De, Bitencourt, G., Hamaji, A., \& Wynne, L. (2020). Since January 2020 Elsevier has created a COVID-19 resource centre with free information in English and Mandarin on the novel coronavirus COVID- 19. The COVID-19 resource centre is hosted on Elsevier Connect, the company ' $\mathrm{s}$ public news and information. January.

Okunlola, A. I. (2021). Awake craniotomy in a Covid-19 positive patient: The challenges and outcome. Interdisciplinary Neurosurgery: Advanced Techniques and Case Management, 24. https://doi.org/10.1016/j.inat.2020.101064

Oliveira, F. J. D. de, Costa, L. L., Sousa, L. C. de, Amancio, A. de M., Silva, G. G. da, Pinheiro, J. C., Silva Filho, P. S. F. da, Brasil, G. M. L. C. ., Jales, J. M. R. ., Oliveira, D. J. D. de, \& Paiva, D. F. F. (2020). Valor prognóstico do SOX2 em Carcinomas Epidermóides de Cabeça e Pescoço: Revisão sistemática. Research, Society and Development, 9(9), e587997722. https://doi.org/10.33448/rsd-v9i9.7722

Ponde, V., Diwan, S., Gopal, T. S., Subramanian, Jb., \& Danish, M. (2020). Regional anesthesia in the coronavirus disease (COVID-19) pandemic: Clinical guidelines by AORA, India. Journal of Anaesthesiology Clinical Pharmacology, 36(5), 109. https://doi.org/10.4103/joacp.JOACP_277_20

Rajput, V. K., Tuvar, S., Bhalsing, S. S. S., \& Bhalsing, S. S. S. (2021). Resurgence of combined intravenous Ketamine and regional anesthesia in pediatric ocular surgery in COVID-19 pandemic. Indian Journal of Ophthalmology, 69(2), 395-399. https://doi.org/10.4103/ijo.IJO_2839_20

Goldhoorn, R., Bernsen, M., Hofmeijer, J., Martens, J., Lingsma, H., Dippel, D., Lugt, A., Buhre, W., Roos, Y., Majoie, C., Vos, J., oiten, J., Bart Emmer, R. J. van O. (n.d.). Anesthetic management during endovascular treatment of acute ischemic stroke in the MR CLEAN registry. Neurology. https://doi.org/10.1212 / WNL.0000000000008674

Romanzi, A, Galletti, M., Macchi, L., Putortì, A., Rossi, F., Scolaro, R., \& Vannelli, A. (2020). Awake laparotomy: is locoregional anesthesia a functional option for major abdominal surgeries in the COVID-19 era? European Review for Medical and Pharmacological Sciences, 24(9), 5162-5166. https://doi.org/10.26355/eurrev_202005_21211

Romanzi, A., Boleso, N., Di Palma, G., La Regina, D., Mongelli, F., Milanesi, M., Putortì, A., Rossi, F., Scolaro, R., Zanardo, M., \& Vannelli, A. (2021). Awake Major Abdominal Surgeries in the COVID-19 Era. Pain Research and Management, 2021, 1-6. https://doi.org/10.1155/2021/8763429

Rossi, G., Ferrari, S. A., Sommaruga, S., Muzzarelli, L., \& Piconi, S. (2020). An infection-oriented approach for thoracic endovascular aortic repair in a SARS-CoV-2 positive patient. A case report. Annali Italiani Di Chirurgia, 91, 273-276. http://www.ncbi.nlm.nih.gov/pubmed/32877380

Sabino-silva, R., Carolina, A., Jardim, G., \& Siqueira, W. L. (2020). Sabino-Silva2020_Article_CoronavirusCOVID-19ImpactsToDe.pdf. 1619-1621.

Sadr, M. A. H., Gardiner, M. S., Burr, M. N., Nikkhah, M. D., \& Jemec, M. B. (2020). Managing Hand Trauma during the COVID-19 pandemic using a OneStop Clinic. Journal of Plastic, Reconstructive \& Aesthetic Surgery, 73(7), 1357-1404. https://doi.org/10.1016/j.bjps.2020.05.026

Saha, S., Dash, S., Ansari, M. T., Bichupuriya, A. D., Gupta, A. K., \& Singhal, M. (2021). Optimising Hand Surgery during COVID-19 Pandemic. The Journal of Hand Surgery (Asian-Pacific Volume), 26(01), 84-91. https://doi.org/10.1142/S2424835521500132

Sarkar, S., Banerji, A., Chattopadhyaya, A., \& Banerjee, S. (2021). Lumbar spine instrumented fusion surgery under spinal anaesthesia versus general anaesthesia-A retrospective study of 239 cases. Journal of Clinical Orthopaedics and Trauma, 18, 205-208. https://doi.org/10.1016/j.jcot.2021.04.026

Shih, M., Swearingen, B., \& Rhee, R. (2020). Ruptured Abdominal Aortic Aneurysm Treated with Endovascular Repair in a Patient with Active COVID-19 Infection during the Pandemic. Annals of Vascular Surgery, 66, 14-17. https://doi.org/10.1016/j.avsg.2020.05.001

Sorbo, G., Gagliano, S., La Tessa, C., Ferrarini, R., Galluzzo, V., \& Cucchi, M. C. (2020). Breast unit Efficacy with the relocation of the surgical breast team during the COVID-19 pandemic. The Breast Journal, 26(8), 1613-1614. https://doi.org/10.1111/tbj.13934 
Thomas, V., Maillard, C., Barnard, A., \& Snyman, L. (2020). Since January 2020 Elsevier has created a COVID-19 resource centre with free information in English and Mandarin on the novel coronavirus COVID- 19. The COVID-19 resource centre is hosted on Elsevier Connect, the company' s public news and information . January.

Tognù, A., Barbara, E., Pacini, I., \& Bosco, M. (2021). Proximal humeral fracture surgery in the COVID-19 pandemic: advocacy for regional anesthesia. Regional Anesthesia \& Pain Medicine, 46(4), 375-376. https://doi.org/10.1136/rapm-2020-101626

Tonyali, S., Haberal, H. B., Ergul, R., \& Dursun, M. (2020). Management of Patients Who Seek Urologic Care in Covid-19 Pandemic Era. Urology Journal, 17(5), 548-554. https://doi.org/10.22037/uj.v16i7.6285

Turcotte, J. J., Petre, B. M., Jones, C. M., \& Gelfand, J. M. (2020). Maintaining Access to Orthopaedic Surgery During Periods of Operating Room Resource Constraint: Expanded Use of Wide-Awake Surgery During the COVID-19 Pandemic. JAAOS: Global Research and Reviews, 4(12), e20.00100. https://doi.org/10.5435/JAAOSGlobal-D-20-00100

Ung, N., \& Bonnet, M. P. (2020). Anesthésie de la femme enceinte dans le contexte de la pandémie de COVID-19. Le Praticien En Anesthésie Réanimation, 24(4), 196-201. https://doi.org/10.1016/j.pratan.2020.07.005

Uppal, V., Sondekoppam, R. V, Landau, R., El-Boghdadly, K., Narouze, S., \& Kalagara, H. K. P. (2020). Neuraxial anaesthesia and peripheral nerve blocks during the COVID-19 pandemic: a literature review and practice recommendations. Anaesthesia, 75(10), 1350-1363. https://doi.org/10.1111/anae.15105

Varandas, J. S., Dias, R., Mendes, A. B., Lages, N., \& Machado, H. (2021). New indication for an old anesthetic technique: could we consider now rapid sequence spinal anesthesia in a COVID-19 time? Regional Anesthesia \& Pain Medicine, 46(2), 191.1-191. https://doi.org/10.1136/rapm-2020-101572

Verikokos, C., Lazaris, A. M., \& Geroulakos, G. (2020). Doing the right thing for the right reason when treating ruptured abdominal aortic aneurysms in the COVID-19 era. Journal of Vascular Surgery, 72(1), 373-374. https://doi.org/10.1016/j.jvs.2020.04.009

Wade, S., Nair, G., Ayeni, H. A., \& Pawa, A. (2020). A Cohort Study of Emergency Surgery Caseload and Regional Anesthesia Provision at a Tertiary UK Hospital During the Initial COVID-19 Pandemic. Cureus, 12(6). https://doi.org/10.7759/cureus.8781

Zhong, Q., Liu, Y. Y., Luo, Q., Zou, Y. F., Jiang, H. X., Li, H., Zhang, J. J., Li, Z., Yang, X., Ma, M., Tang, L. J., Chen, Y. Y., Zheng, F., Ke, J. J., \& Zhang, Z. Z. (2020). Spinal anaesthesia for patients with coronavirus disease 2019 and possible transmission rates in anaesthetists: retrospective, single-centre, observational cohort study. British Journal of Anaesthesia, 124(6), 670-675. https://doi.org/10.1016/j.bja.2020.03.007 